\title{
Anti-TNF therapy in the management of ocular attacks in an elderly patient with long-standing Behçet's disease
}

\author{
Hisako Karube \\ Koju Kamoi \\ Kyoko Ohno-Matsui
}

Department of Ophthalmology and Visual Science, Graduate School of Medical and Dental Sciences, Tokyo Medical and Dental University, Tokyo, Japan
Correspondence: Koju Kamoi

Department of Ophthalmology and Visual Science, Graduate School of Medical and Dental Sciences, Tokyo Medical and Dental University, I-5-45 Yushima, Bunkyo-ku, Tokyo II 3-85 I9, Japan

$\mathrm{Tel}+81358035302$

Fax +8I 338187188

Email koju.oph@tmd.ac.jp
This article was published in the following Dove Press journal:

International Medical Case Reports Journal

27 September 2016

Number of times this article has been viewed

Background: Ocular symptoms in Behçet's disease (BD) begin mostly before 30 years of age according to international surveys, and $\mathrm{BD}$ activity may decrease with age. Information regarding the treatment of ocular symptoms in elderly BD patients is thus scant. Anti-TNF $\alpha$ antibody has recently demonstrated strong effects against recurrent uveitis in $\mathrm{BD}$, but the efficacy and safety of anti-TNF $\alpha$ therapy in elderly patients remain unclear. We report herein the case of an elderly patient with long-standing uveitis due to BD who was successfully treated with two types of anti-TNF therapy.

Case: An 81-year-old Japanese man presented with a 33-year history of ocular inflammation due to BD. As immunosuppressive agents, such as cyclosporine A, were difficult to use because he had undergone removal of the left kidney due to cancer, he was treated with colchicine. However, attacks of ocular inflammation persisted around nine times a year. After colchicine had been changed to infliximab, ocular inflammation was fairly well controlled, but ocular attacks still occurred once or twice a year. As soon as intestinal hemorrhage related to BD occurred, infliximab was switched to adalimumab. After this switch, ocular attacks resolved and visual acuity was maintained at 1.0. Intestinal lesions were also well controlled, and no side effects were seen. Conclusion: This represents the first report of the application of two types of anti-TNF $\alpha$ therapy for ocular attacks in an elderly BD patient. In addition to infliximab, adalimumab appears to offer an alternative therapy for refractory, long-standing BD-related uveitis in elderly patients. Keywords: Behçet's disease, anti-tumor necrosis factor- $\alpha$ antibody, infliximab, adalimumab, elderly patient

\section{Background}

Behçet's disease (BD) is a systemic inflammatory disease characterized by recurrent ocular inflammation, oral and mucosal ulcers and skin lesions, and other manifestations in multiple organ systems. Colchicine, cyclosporine, corticosteroids, azathioprine, and other immunosuppressive agents are used to treat attacks of ocular inflammation in BD. ${ }^{1}$

Ocular inflammatory attacks in BD have been reported to begin before 30 years of age according to international surveys. ${ }^{2}$ One reason may be that BD activity decreases with patient age, and little information is available regarding the treatment of elderly BD patients.

As recent treatments for $\mathrm{BD}$, infliximab, a chimeric human murine monoclonal TNF $\alpha$ antibody, has demonstrated marked effects for controlling uveitis in BD. However, infliximab requires intravenous infusion, and infusion reactions represent a known adverse effect. Adalimumab is another TNF $\alpha$ blocker, representing a fully human monoclonal anti-TNF $\alpha$ antibody that can be self-administered subcutaneously. While adalimumab is currently used for ankylosing spondylitis, Crohn's disease, juvenile idiopathic arthritis, 
plaque psoriasis, psoriatic arthritis, rheumatoid arthritis, and ulcerative colitis, this agent has not been used for ocular inflammation as an approved drug. Reports have established adalimumab as an efficacious and safe treatment in patients with Crohn's disease, rheumatoid arthritis, and psoriasis. ${ }^{3-5}$ However, whether these anti-TNF $\alpha$ therapies are effective and safe for elderly BD patients with ocular inflammation remains unclear.

We report herein the case of an elderly patient with a 33-year history of BD in whom disease control with standard treatments was proving difficult, but ocular inflammation was completely suppressed after switching to infliximab and adalimumab. The patient provided written informed consent for the publication of their data and accompanying images.

\section{Case presentation}

An 81-year-old Japanese man was diagnosed with BD in 1981 on the basis of recurrent oral and genital ulcers, erythema nodosum, and uveitis, meeting the classification criteria for BD established by the Japanese Ministry of Health and Welfare. ${ }^{6}$ He lost eyesight in his left eye in 1992, due to recurrent uveitis. He was referred to our hospital in 2007 with recurrent attacks of inflammation affecting the right eye. He had been treated with betamethasone eyedrops, but had never been treated with colchicine or cyclosporine A before the first visit to our hospital. As for initial ophthalmic presentation at our hospital, visual acuity in the right eye was at the level of recognizing hand motion, and keratic precipitate, anterior chamber cells $(++)$, flare $(++)$, and grade 3 vitreous haze were identified. The left eye showed no inflammation, and light perception on electroretinography was unrecordable.

The patient was initially treated with colchicine and betamethasone eyedrops, but attacks of ocular inflammation persisted, occurring six times in 8 months (equivalent to nine times/year). Immunosuppressive agents, such as cyclosporine A, were difficult to use because he had undergone removal of the left kidney due to cancer in 1998. In May 2008, administration of infliximab was started at a dose of $5 \mathrm{mg} / \mathrm{kg}$, followed by a regular treatment schedule (initially in weeks 2 and 6 , then every 8 weeks). This treatment reduced the frequency of ocular inflammatory attacks to seven times in 60 months (1.4 times/year). Right visual acuity improved to 1.0. However, the patient experienced exanthema after the ninth infusion of infliximab in May 2009 (54 weeks after starting infliximab treatment). That episode was considered to represent an infusion reaction to infliximab, so $5 \mathrm{mg}$ of D-chlorpheniramine maleate and $20 \mathrm{mg}$ of epinastine hydrochloride were administered before each infusion of infliximab thereafter.

The frequency of ocular attacks increased from December 2011 ( 3 years and 7 months after starting infliximab treatment), and oral ulcers and abdominal pain occurred in the same period. After 4 years and 8 months, intestinal lesions with bleeding were identified, and infliximab was judged inadequate to control intestinal lesions. Adalimumab was thus considered as an alternative treatment, and started as a substitute for infliximab. Adalimumab was administered at a dose of $80 \mathrm{mg}$ the first time, with $40 \mathrm{mg}$ administered every 2 weeks thereafter. Adalimumab was self-administered subcutaneously by the patient. Visual acuity prior to the initiation of adalimumab was 1.0. After switching to adalimumab, uveitis was well controlled, right visual acuity remained at 1.0 , and ocular inflammatory attacks did not occur during 2 years of follow-up (Figure 1).

Vitreous haze in the right eye had disappeared with the use of infliximab, and did not reappear after switching to

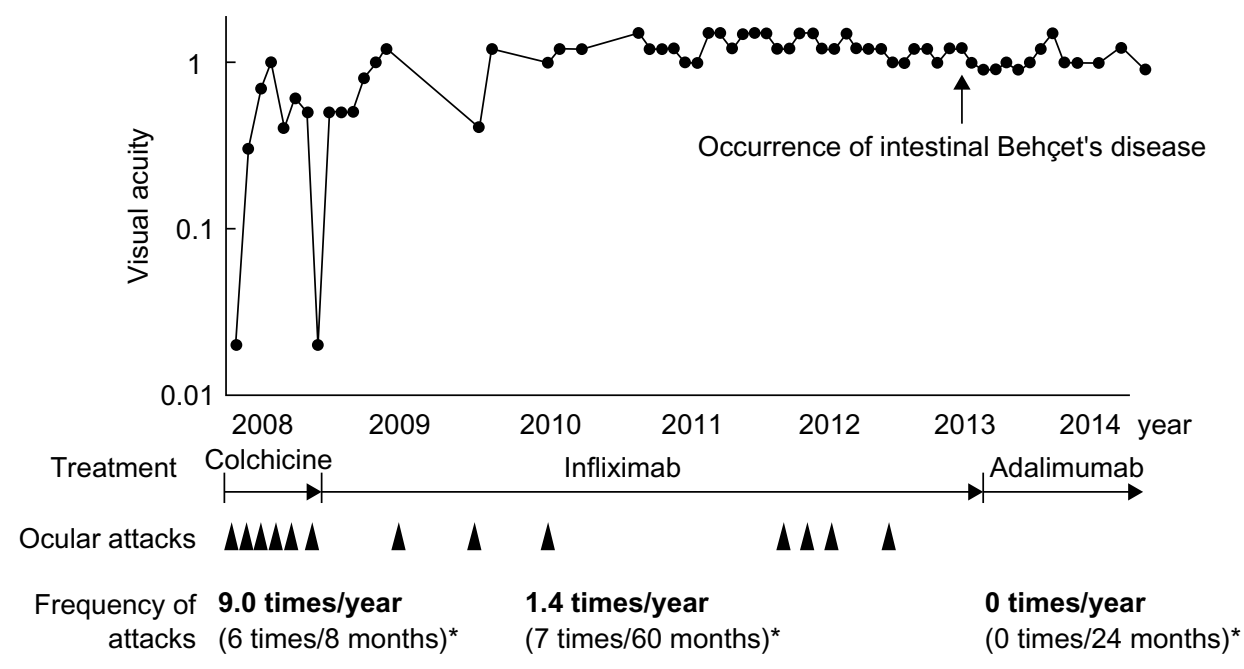

Figure I Changes in right visual acuity and frequency of ocular attacks during treatment.

Notes: Visual acuity remained at I.0, and no ocular attacks occurred after switching to adalimumab. Black arrowheads indicate the timing of ocular attacks. ${ }^{*}$ The frequency of attacks was converted into an annual frequency. Data in parentheses indicates the actual frequency of attacks during representative treatments. 

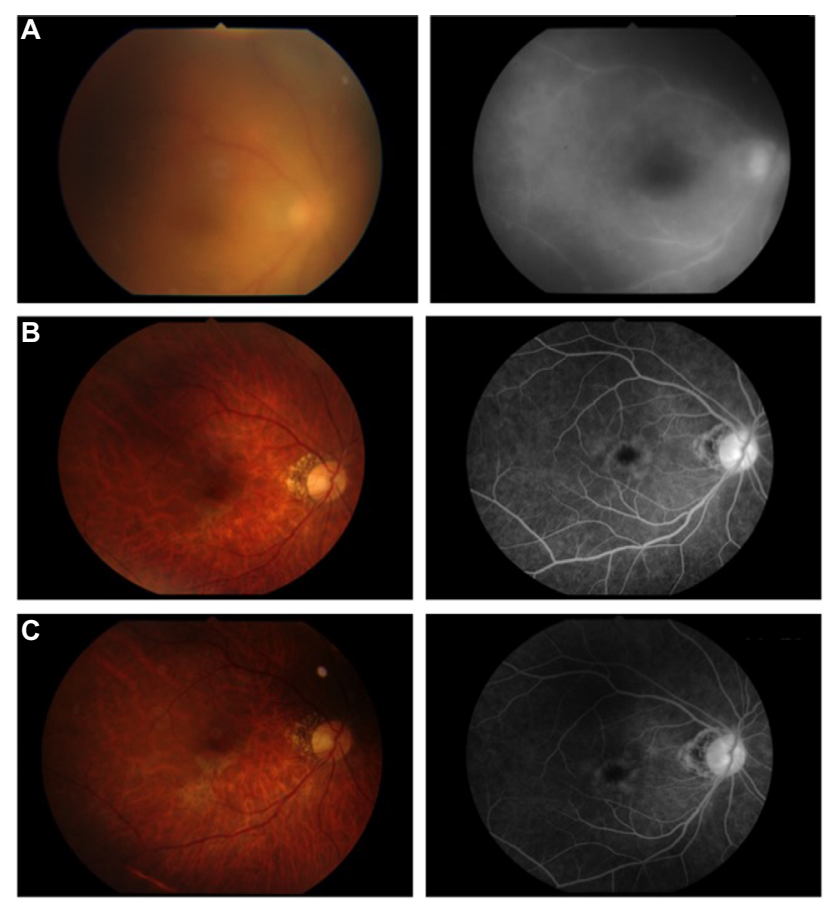

Figure 2 Fundus color photograph (left) and fluorescein angiography (right) of the right eye.

Notes: (A) Initial presentation. (B) Three years and 7 months after starting infliximab. (C) Five months after starting adalimumab. Vitreous haze was apparent at the first examination (A, left), but disappeared after starting infliximab (B, left) and did not reappear after switching to adalimumab (C, left). Leakage from retinal vessels was seen in fluorescein angiography examination before treatment (A, right), but improved with infliximab (B, right) and adalimumab (C, right).

adalimumab. Fluorescein angiography examination showed leakage from retinal vessels at the first examination, but this leakage was dramatically improved by infliximab, and the improvement was maintained on adalimumab (Figure 2). Intestinal lesions were also well controlled.

\section{Conclusion}

Research into the application of anti-TNF $\alpha$ to certain diseases, such as rheumatoid arthritis and bowel disease, has reported that elderly patients receiving anti-TNFo show slightly dampened responses compared with younger patients, although once an initial response is achieved, longterm outcomes are similar. In terms of the risk of anti-TNF $\alpha$, elderly patients receiving anti-TNF present with an increased risk of adverse events, such as serious and opportunistic infections, compared with younger patients. Such risk varies according to the type of adverse event when elderly patients receive other treatments. Comorbidities, such as cardiovascular disease, are an important element to consider in elderly patients. ${ }^{7,8}$

In elderly BD patients, the efficacy and safety of antiTNF $\alpha$ have remained unclear because of the lack of published data. One reason for this lack may involve a proposed self-limiting mechanism of ocular attacks with increasing age in $\mathrm{BD}$, and anti-TNF $\alpha$ agents have thus remained largely unnecessary for elderly BD patients. We encountered a rare case of $\mathrm{BD}$ in which the patient continued suffering from frequent ocular uveitis attacks even in older age, and administration of anti-TNF therapy proved useful.

In the present case, changing from colchicine to infliximab was obviously successful, because the frequency of ocular attacks fell from nine times a year to once or twice a year. After starting infliximab, visual acuity gradually improved. Infliximab had achieved good control of the uveitis, but the frequency of ocular attacks increased after 3 years and 7 months (in December 2011). Treatment with infliximab can result in immunogenicity and the formation of human antichimeric antibodies (HACAs), which act as antibodies to infliximab. The appearance of HACAs has been found in more than half of patients who have received infliximab. ${ }^{9}$ The formation of HACAs was the most likely reason for the increase in ocular attacks in this case. Infliximab was used for 4 years and 8 months, and the course suggests the development of HACAs, resulting in inflammation of the intestinal tract. Changing to another anti-TNF $\alpha$ agent proved effective, and was considered a good alternative.

From a pharmacokinetic perspective, subcutaneous administration of adalimumab leads to more uniform serum concentrations at a steady state, whereas intravenous administration of infliximab leads to higher peak and lower trough levels. These pharmacokinetic differences may result in different safety and efficacy profiles. However, the effectiveness and safety outcomes are reportedly comparable between intravenous and subcutaneous administration of anti-TNF $\alpha$ agents. ${ }^{10}$

In this case, after a switch from infliximab to adalimumab, ocular and intestinal symptoms were not encountered for more than 2 years, and no adverse effects have been seen. Adalimumab was obviously able to prevent long-standing recurrent ocular inflammation in this elderly patient.

Investigations into noninfectious uveitis have shown the mean time to discontinuation of infliximab secondary following development of rash was 39.8 weeks, and the mean time to elevated liver function test results was 54 weeks. Infusion-related reaction occurred at a mean of 35.2 weeks. Differences in these durations by age have not been clarified. ${ }^{11}$ The patient developed exanthema after the ninth infusion of infliximab (54 weeks after starting infliximab), which was considered to represent an infusion reaction to infliximab and occurred after a longer-than-average duration. With the switch to adalimumab, no adverse effects, eg, infusion 
reactions, were encountered. As adalimumab represents a fully human monoclonal anti-TNF $\alpha$ antibody, whereas infliximab is a chimeric human murine monoclonal antibody, the risk of infusion reactions may be reduced with adalimumab.

Data concerning the efficacy of adalimumab for BDrelated uveitis in elderly patients are limited, although several reports have shown that adalimumab therapy for refractory noninfectious uveitis is useful for patients of susceptible ages, ${ }^{12-16}$ and outcomes in terms of safety appear superior to those of infliximab. ${ }^{12}$ Accumulation of long-term followup data from elderly patients with active BD is needed to evaluate the length of remission that can be achieved, but adalimumab appears to offer a feasible alternative treatment for BD-related uveitis in elderly patients.

This case, with marked suppression of ocular inflammation using adalimumab without side effects in an elderly patient with long-standing BD, may provide good information for ophthalmologists.

\section{Disclosure}

The authors report no conflicts of interest in this work.

\section{References}

1. Evereklioglu C. Current concepts in the etiology and treatment of Behçet disease. Surv Ophthalmol. 2005;50(4):297-350.

2. Kitaichi N, Miyazaki A, Iwata D, Ohno S, Stanford MR, Chams H. Ocular features of Behçet's disease: an international collaborative study. Br J Ophthalmol. 2007;91(12):1579-1582.

3. Da W, Zhu J, Wang L, Lu Y. Adalimumab for Crohn's disease after infliximab treatment failure: a systematic review. Eur J Gastroenterol Hepatol. 2013;25(8):885-891.
4. Smolen JS, Emery P, Fleischmann R, et al. Adjustment of therapy in rheumatoid arthritis on the basis of achievement of stable low disease activity with adalimumab plus methotrexate or methotrexate alone: the randomised controlled OPTIMA trial. Lancet. 2014;383(9914):321-332.

5. Leonardi C, Papp K, Strober B, et al. The long-term safety of adalimumab treatment in moderate to severe psoriasis: a comprehensive analysis of all adalimumab exposure in all clinical trials. Am J Clin Dermatol. 2011;12(5):321-337.

6. Behçet's Disease Research Committee of Japan. Behçet's disease: guide to diagnosis of Behçet's disease. Jpn J Ophthalmol. 1974;18:291-294.

7. Lahaye C, Tatar Z, Dubost JJ, Soubrier M. Overview of biologic treatments in the elderly. Joint Bone Spine. 2015;82(3):154-160.

8. Lobatón T, Ferrante M, Rutgeerts P, Ballet V, Van Assche G, Vermeire S. Efficacy and safety of anti-TNF therapy in elderly patients with inflammatory bowel disease. Aliment Pharmacol Ther. 2015;42(4):441-451.

9. Baert F, Noman M, Vermeire S, et al. Influence of immunogenicity on the long-term efficacy of infliximab in Crohn's disease. $N$ Engl J Med. 2003;348(7):601-608.

10. Liu J, Sylwestrzak G, Ruggieri AP, DeVries A. Intravenous versus subcutaneous anti-TNF-alpha agents for Crohn's disease: a comparison of effectiveness and safety. J Manag Care Spec Pharm. 2015;21(7): 559-566.

11. Kruh JN, Yang P, Suelves AM, Foster CS. Infliximab for the treatment of refractory noninfectious uveitis: a study of 88 patients with long-term follow-up. Ophthalmology. 2014;121(1):358-364.

12. Suhler EB, Lowder CY, Goldstein DA, et al. Adalimumab therapy for refractory uveitis: results of a multicentre, open-label, prospective trial. Br J Ophthalmol. 2013;97(4):481-486.

13. Díaz-Llopis M, Salom D, Garcia-de-Vicuña C, et al. Treatment of refractory uveitis with adalimumab: a prospective multicenter study of 131 patients. Ophthalmology. 2012;119(8):1575-1581.

14. Perra D, Alba MA, Callejas JL, et al. Adalimumab for the treatment of Behçet's disease: experience in 19 patients. Rheumatology (Oxford). 2012; 51(10): 1825-1831.

15. Takase K, Ohno S, Ideguchi H, Uchio E, Takeno M, Ishigatsubo Y. Successful switching to adalimumab in an infliximab-allergic patient with severe Behçet disease-related uveitis. Rheumatol Int. 2011;31(2): 243-245.

16. Mushtaq B, Saeed T, Situnayake RD, Murray PI. Adalimumab for sightthreatening uveitis in Behçet's disease. Eye (Lond). 2007;21(6):824-825.
International Medical Case Reports Journal

\section{Publish your work in this journal}

The International Medical Case Reports Journal is an international, peer-reviewed open-access journal publishing original case reports from all medical specialties. Previously unpublished medical posters are also accepted relating to any area of clinical or preclinical science. Submissions should not normally exceed 2,000 words or

\section{Dovepress}

4 published pages including figures, diagrams and references. The manuscript management system is completely online and includes a very quick and fair peer-review system, which is all easy to use. Visit $\mathrm{http}: / /$ www.dovepress.com/testimonials.php to read real quotes from published authors. 